\title{
"Ultrasongraphic And Colour Doppler Evaluation of Cervical Lymphadenopathy with fine Needle Aspiration Cytology Correlation."
}

\author{
${ }^{1}$ Dr. Awdhut Tiparse, ${ }^{1}$ Dr. Birwa Gandhi,${ }^{1}$ Dr. Arpita Patel,,${ }^{1}$ Dr. Aastha Bhatt. \\ ${ }^{1}$ Resident doctors,Department of Radio diagnosis, Government medical college, Bhavnagar, Gujrat, India. \\ ${ }^{2}$ Department of Radio diagnosis. Government medical college, Sir T Hospital, Bhavnagar
}

Gujrat ,India

\begin{abstract}
:
Introduction: Cervical lymphadenopathy is one of the most common causes of mass in head and neck region. Patients either present with palpable neck masses or with malignancy of the head and neck where the preoperative detection of the involved nodes is essential. Ultrasound and color/power Doppler are used to localize the node and evaluate their multiplicity, size, shape (roundness index), hilum status, margin, calcification, necrosis (>3 mm), and peri lymphnodal edema. Color and power Doppler is used to know the pattern of vascularity and doppler indices [resistive index (RI) and pulsatility index (PI)].

Aims and Objectives: The aims and objectives of this study were to study and differentiate between neoplastic (malignant) and non neoplastic (reactive and tubercular) cervical lymph nodes by high-resolution ultrasonography. To correlate between ultrasound and fine needle aspiration cytology (FNAC) in cervical lymphadenopathy.

Material and Methods: Data were collected from a total of 160 cases referred for an ultrasound of neck to the Department of Radio diagnosis, Government medical college and Sir T Hospital, Bhavnagar, from December 2017 to June 2017, with Esaote Mylab 40 ultrasound machine. Lymph nodes were assessed using gray scale and color Doppler parameters such as nodal level and site, size, shape, L/S ratio, border, hilum, echo texture, necrosis, matting,. A provisional diagnosis was suggested after the ultrasound examination, and these findings were correlated with FNAC findings.

Results: In our study160 patients, out of 95 non-neoplastic nodes (reactive and tubercular), only 80 nodes were identified as non-neoplastic (reactive/tubercular) on ultrasound prior to FNAC/histopathology. Out of 70 possible neoplastic (malignant nodes) detected on ultrasound, only 58 lymph nodes turned out to be neoplastic on FNAC/histopathology. Lymph node with oval shape (L/S ratio >2) echogenic hilum, homogenous echo texture, and hilar vascularity was considered as significant parameters in detecting non-neoplastic (reactive) lymph nodes, which showed matting with soft tissue edema. Correlation of sonographic findings with FNAC findings was performed. Sensitivity and specificity of ultrasound in differentiating neoplastic from nonneoplastic cervical lymphadenopathy was found to be $90 \%$ and $76 \%$, respectively.

Conclusion: We conclude that ultrasonographic examination of cervical lymph nodes can yield important information regarding the diagnosis. The sonographic features should be used in conjunction with FNAC findings and may be especially helpful in cytologically indeterminate cases. Ultrasound examination should be done prior to FNAC and ideally an ultrasound guided FNAC sample should be obtained from the most sonographically representative node to reduce the sampling error. A lymph node biopsy can often be avoided by utilizing a combination of FNAC and ultrasonographic examination of the neck node.
\end{abstract}

\section{Introduction}

Cervical lymphadenopathy is one of the most common causes of mass in head and neck region; there are various causes of CL common among them are reactive, tuberculosis, metastasis, and lymphoma. Ultrasonographic criteria for distinguishing neoplastic and non-neoplastic lymph nodes have been studied under site, shape, size, echogenicity, hilum, matting, nodal border, long/short axis ratio, intra nodal necrosis, and angioarchitecture. ${ }^{1}$ Ultrasonographic features that help to identify abnormal nodes as well as giving clues to neoplastic nodes are heterogeneous echogenicity, absent hilus, invasion, and intra nodal necrosis. The shape is the best method to attempt the differentiation between neoplastic and non-neoplastic lymph nodes. The long/short diameter ratio of lymph node provides excellent criteria for differentiation between neoplastic and non-neoplastic cervical lymphadenopathy. ${ }^{2}$ 


\section{Material And Method}

The study was conducted at Department of Radio diagnosis, Government medical college and Sir T Hospital, Bhavnagar, from December 2017 to June 2017 with Esaote My lab 40 ultrasound machine. The Data were collected from a total of 160 cases referred for an ultrasound of neck for neck swelling or suspected cervical lymphadenopathy.

\section{Inclusion Criteria:}

1. All patients coming for ultrasound neck for neck swelling.

2. Patients of age group 10-12 years of age of either sex.

3. Patients giving consent for FNAC.

\section{Exclusion Criteria:}

1.Patients who are not giving consent for USG and FNAC examination.

2.Moribund patients.

3. Patient with no evidence of cervical lymphadenopathy on ultrasound.

The criteria that are followed in this study to differentiate between reactive, tubercular, and neoplastic (malignant) lymph nodes: 1. Distribution includes levels and side; 2. Number; 3. Size; 4. Shape includes L/S ratio; 5. Echogenic hilum - wide, narrow, and absent. Nodes hypoechoic, round without echogenic hilus, intranodal cystic necrosis, nodal matting, and adjacent soft tissue edema were considered tubercular lymphadenitis. Round shape, absent hilum, heterogenous echotexture, sharp borders, L/S ratio. Non-neoplastic lymph nodes include reactive and tubercular. Lymph node oval shape, echogenic hilum, homogenous echo texture, matting, L/S ratio $>2$, and hilar vascularity were considered as reactive lymphadenopathy. The most promising contribution of ultrasound is in the guidance of FNAC in non-palpable lymph nodes. Under aseptic precaution and ultrasound guidance, $21 / 22$ gauge needle with syringe is introduced into enlarged abnormal lymph nodes, and sample is sent for analysis.

\section{Result}

In our study of 160 patients, Patients from all age group of either sex from 10 to 80 years were included.

Table: 1 Age Distrubtion of Patients:

\begin{tabular}{|l|l|l|}
\hline Age group in years & Number of cases $(\mathbf{n}-160)$ & Percentage $\%$ \\
\hline $10-20$ & 37 & 23.12 \\
\hline $21-30$ & 23 & 14.3 \\
\hline $31-40$ & 22 & 13.7 \\
\hline $41-50$ & 28 & 17.5 \\
\hline $51-60$ & 26 & 16.2 \\
\hline $61-70$ & 16 & 10 \\
\hline $71-80$ & 18 & 11.2 \\
\hline$>80$ & 10 & 6.25 \\
\hline Total & 160 & \\
\hline
\end{tabular}

Most common age group was 10-20 (23.12\%) years and least common age group was >80 years (6.25).

Table :2 Ultrasonographic evaluation of cervical lymphadenopathy.

\begin{tabular}{|l|l|l|}
\hline USG Diagnosis & Number of cases ( n-160) & Percentage\% \\
\hline Malignant & 70 & 43.7 \\
\hline Tubercular & 42 & 26.5 \\
\hline Reactive & 48 & 30 \\
\hline Total & 160 & \\
\hline
\end{tabular}

Most common ultrasongraphic diagnosis was Malignant lymphnodes (43.7 \%) followed by reactive lymphnode (30\%)and tubercular( $42 \%)$.

Table : 3 Fine needle aspiration cytology diagnosis.

\begin{tabular}{|l|l|l|}
\hline FNAC Diagnosis & Number of cases (n-160) & Percentage\% \\
\hline Malignant & 58 & 36.25 \\
\hline Tubercular & 42 & 26.25 \\
\hline Reactive & 60 & 37.5 \\
\hline Total & 160 & \\
\hline
\end{tabular}

In present study, out of 90 non neoplastic lymphnodes, only 80 were identified as non neoplastic (tubercular/ reactive) and out of 70 possible neoplastic nodes detected by ultrasound 58 were lymphnodes were turned to be neoplastic on FNAC. 
Table 4: Comparison of USG diagnosis with FNAC correlation.

\begin{tabular}{|l|l|l|}
\hline Diagnosis & Ultrasound diagnosis & FNAC diagnosis \\
\hline Malignant & $70(43.7 \%)$ & $58(36.25 \%)$ \\
\hline Tubercular & $42(26.5 \%)$ & $42(26.5 \%)$ \\
\hline Reactive & $48(30 \%)$ & $60(37.5 \%)$ \\
\hline Total & 160 & 160 \\
\hline
\end{tabular}

Table 5: Hilar vascularity on USG colour doppler diagnosis with FNAC diagnosis.

\begin{tabular}{|l|l|l|l|}
\hline Diagnosis & Present (\%) & Absent(\%) & Total \\
\hline Malignant & $14(24 \%)$ & $44(75 \%)$ & 56 \\
\hline Tubercular & $6(14 \%)$ & $36(85 \%)$ & 42 \\
\hline Reactive & $44(73 \%)$ & $16(26 \%)$ & 60 \\
\hline Total & $64(40 \%)$ & $96(60 \%)$ & 160 \\
\hline
\end{tabular}

Most common lymhnodes showing hilar vascularity was reactive lymphnodes (73\%) and Tubercular lymphnodes showed least hilar vascularity (14\%).

Table 6: Capsular vascularity on colour doppler with capsular vascularity in FNAC.

\begin{tabular}{|l|l|l|l|}
\hline Diagnosis & Present (\%) & Absent (\%) & Total \\
\hline Malignant & $6(20 \%)$ & $52(89 \%)$ & 54 \\
\hline Tubercular & $2(9.4 \%)$ & $40(95 \%)$ & 42 \\
\hline Reactive & $6(10 \%)$ & $54(90 \%)$ & 60 \\
\hline Total & $14(17.4 \%)$ & $146(91.2 \%)$ & 160 \\
\hline
\end{tabular}

Capsular vascularity most commonly is seen in malignant lymphnodes (20\%) followed by reactive lymph node $(10 \%)$.

In our study of 160 patients, correlation of sonographic findings with FNAC was performed . sensitivity and specificity of ultrasound in differentiating neoplastic from non neoplastic cervical lymphadenopathy was found to be $90 \%$ and $74 \%$ respectively. Our study had a high sensitivity of $91.3 \%$, specificity of $75.93 \%$,positive predictive value of $(91.11 \%)$ and negative predictive value $76.6 \%$ in differentiating neoplastic from non neoplastic criteria.

\section{Discussion}

Differentiation between tubercular, metastatic, reactive and lymphomatous cervical lymph nodes is extremely important from the therapeutic viewpoint. It is also important to make the correct diagnosis at the earliest because a delayed diagnosis can lead to upstaging of the malignancy making a curable lesion incurable. Clinicians have traditionally relied on FNAC to achieve a tissue diagnosis in cervical lymphadenopathy. The reported sensitivity and specificity of FNAC in the evaluation of cervical lymph nodes are $82 \%$ and $97 \%$ respectively ${ }^{3}$.

However the FNAC report is frequently equivocal. Tubercular lymph nodes may be labeled as reactive or granulomatous lymphadenitis, which puts the treating doctor in a dilemma regarding starting anti tubercular treatment. Similarly in metastatic lymph nodes, sampling errors might occur because the lymph node chosen for FNAC may be reactive while the secondary deposit is harbored by other lymph nodes. Also FNAC is unreliable in differentiating between a metastatic and lymphomatous lymph node. Core needle biopsy is difficult to obtain from cervical lymph nodes. This is because of their small size, typically less than $1.5 \mathrm{~cm}$. Trying to obtain a core needle biopsy especially with a tru cut needle from such small nodes puts the underlying vascular structures at risk of injury. The present study demonstrates the usefulness of ultrasonography used as an adjunct to FNAC in diagnosis of cervical lymphadenopathy.

The ultrasonographic characteristics of tubercular lymphnode are said to be multiple lymph nodes, fusion tendency of adjacent nodes and a hypoechoic center with posterior enhancement. An additional feature, which has great specificity for tubercular lymphadenitis is strong echoes within the mass. The strong echoes are calcification within the node ${ }^{4,5}$. We found strong internal echoes in $84 \%(52 / 62)$ of tubercular lymphnodes and $11 \%(2 / 18)$ of metastatic lymph nodes. Internal echoes were absent in lymphomatous nodes.

Metastatic nodes are ultrasonographically characterized by a smaller long axis to short axis ratio (L/S ratio), absence of hilus and a hypoechoic center. We found that the mean L/S ratio of metastatic nodes was $1.2 \pm$ 0.3 , of lymphomatous nodes $1.5 \pm 0.4$ and tubercular lymph nodes $1.8 \pm 0.6$. An absent hilus was found in $83 \%$ $(15 / 18)$ of metastatic nodes while only $26 \%$ (16/62) of tubercular and $28 \%(4 / 14)$ of lymphomatous nodes had absent hilus. This was because metastatic nodes tend to assume a more spherical shape. Steinkamp HJ et al report that $95 \%$ of metastatic nodes had L/S ratio of less than $2^{6}$. We also found a hypoechoic center in $61 \%$ $(11 / 18)$ of metastatic lymph nodes which could reflect central necrosis. Fusion tendency was found in $66 \%$ $(12 / 18)$ of metastatic nodes which could denote extra nodal spread and should be considered as a prognostic sign and also the need for post surgery adjuvant radiotherapy. Kim $\mathrm{HC}$ et al report the usefulness of $3 \mathrm{D}$ 
ultrasonography for measuring volume of cervical lymph nodes. They found that a cut off volume of $0.7 \mathrm{~cm}^{3} \mathrm{had}$ a $80 \%$ sensitivity and $90 \%$ specificity for differentiating metastatic from reactive lymphadenopathy ${ }^{7}$.Doppler ultrasonography can evaluate the vascular pattern, displacement of vascularity, vascular resistance and pulsatility index. These features have been reported to have a sensitivity of $88 \%$ for the diagnosis of metastatic nodes and $67 \%$ for lymphoma with a specificity of $100 \%{ }^{8}$. Metastatic lymph nodes are reported to have higher resistivity index $(>0.8)$ and pulsatility index $(>1.5)$ than reactive lymph nodes ${ }^{9}$. The limiting feature of Doppler and power ultrasound studies is their inability to distinguish between inflammatory and neoplastic nodes reliably on the basis of their flow pattern. Both metastatic and inflammatory nodes have associated vascularisation, which could appear similar on Doppler scan.

The main distinguishing feature of lymph nodes in lymphoma was a homogeneous pattern. In our study on 14 patients with lymphoma the mean L/S ratio was $1.5 \pm 0.4$, regular margin was seen in $79 \%(11 / 14)$ and none of them showed internal echoes within the lymph node. This could be attributed to the fleshy nature of these nodes and absence of either calcification or necrosis within them ${ }^{10}$.

\section{Comparision With Other Studies:}

In a study done by Danninger et al ultrasonography sensitivity and specifi city for detecting malignant nodes was $96 \%$ and $69 \%$, respectively. ${ }^{11}$ Ahuja and Ying.6 concluded that ultrasound was $95 \%$ sensitive and $83 \%$ specifi c for classifying metastatic/nonmetastatic lymph nodes ${ }^{12}$.

In our present study on USG out of 160 lymph nodes, 75 were malignant, 42 were tubercular and 48 were reactive lymph nodes. On FNAC out of 160 lymph nodes, 58 lymph nodes were malignant, 42 were tubercular, and 60 were reactive lymph nodes (Table 3).In our study, the ultrasonography sensitivity, specificity, positive and negative predictive values are $90 \%, 74 \%, 77 \%$ and $92 \%$, respectively, for differentiating neoplastic from non-neoplastic cervical lymphadenopathy. In a study done by Na et al.9 $97 \%$ of benign/reactive and $18 \%$ of malignant lymph nodes showed hilar vessels. ${ }^{13}$ In our study of 160 lymph nodes: Malignant 14, tubercular 6, and reactive 42 showed hilar vessels. The $\mathrm{P}$ value for this criterion was present study $<0.01$ which showed association very significant. In a study done by $\mathrm{Na}$ et $\mathrm{al}^{13}$ there is peripheral vascularity with the loss of central nodal vascularity is tubercular nodes 24 and metastatic. ${ }^{14}$ Our study shows tubercular 14 and malignant 6 lymph nodes showed only capsular vascularity which was statistically not significant (Table 6). According to Na et al. ${ }^{13}$ it is very difficult to detect superficially located, slow flow signals, It is difficult to obtain Doppler spectral wave forms in non-cooperative patients.

\section{Concluision}

Ultrasonography is cost-effective, easily available, radiation free, non-invasive, safe and is primary investigation to differentiate malignant, tubercular, and reactive cervical lymphadenopathy. Ultrasound examination of the lymph nodes can be done in all planes so that exact nodal size and shape can be evaluated. Ultrasound evaluation is very sensitive in differentiating between cystic/necrotic foci and solid swellings. .Ultrasound helps in identifying abnormal nodes and useful for guided FNAC. . Finally, all ultrasound diagnosis must be correlated with FNAC/histopathology study not only to determine whether the nodes are malignant, reactive, tubercular, nodes but also to determine the histology of the neoplasm.

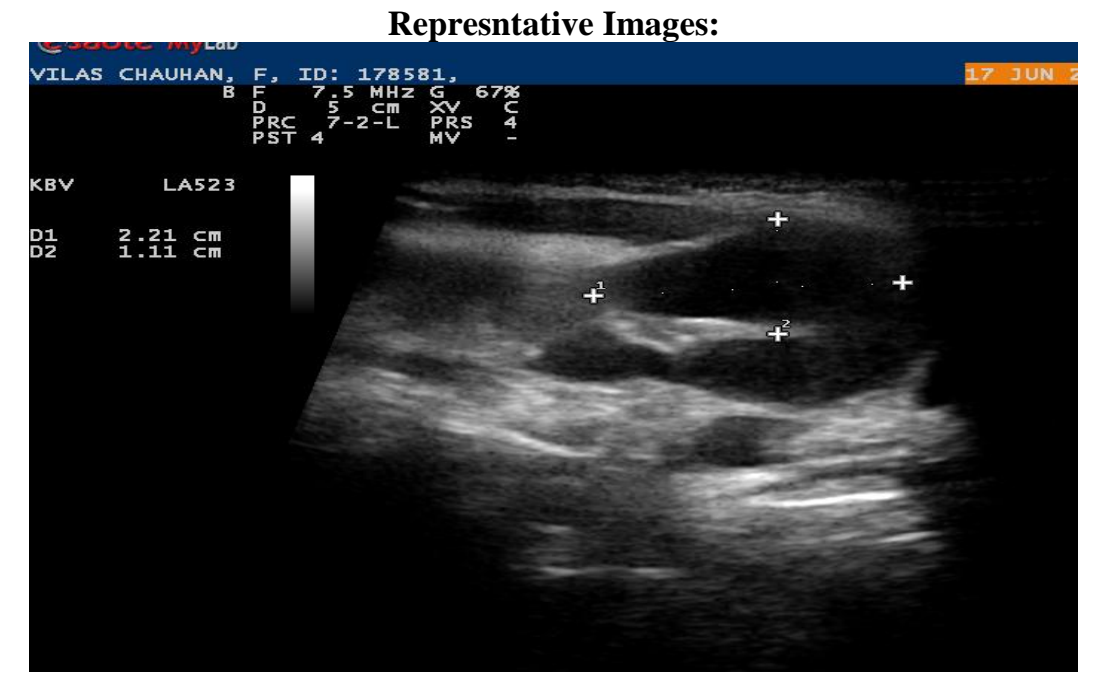

Figure:1 Well defined hypoechoic lesions (reactive lymphnodes) in right side of neck. FNAC proved the Final Diagnosis. 


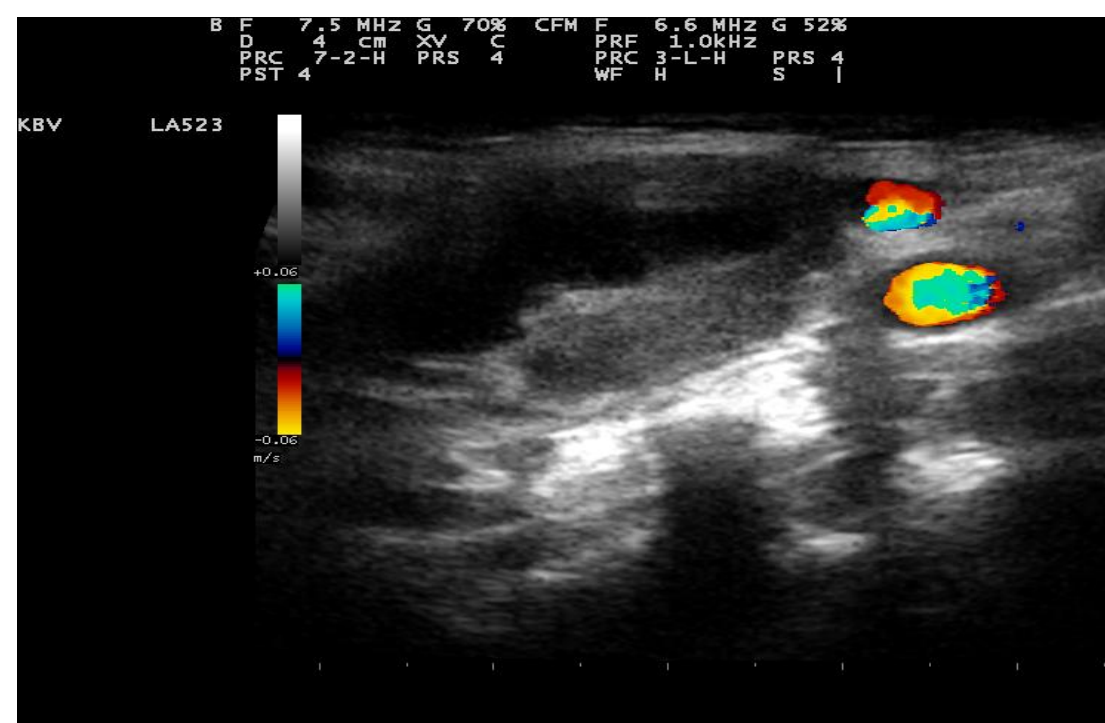

Figure 2: 19 year old male patient known case of Koch's showed hypoechoic lesions with central necrosis in right side (tubercular lymhnodes). FNAC proved the final diagnosis.

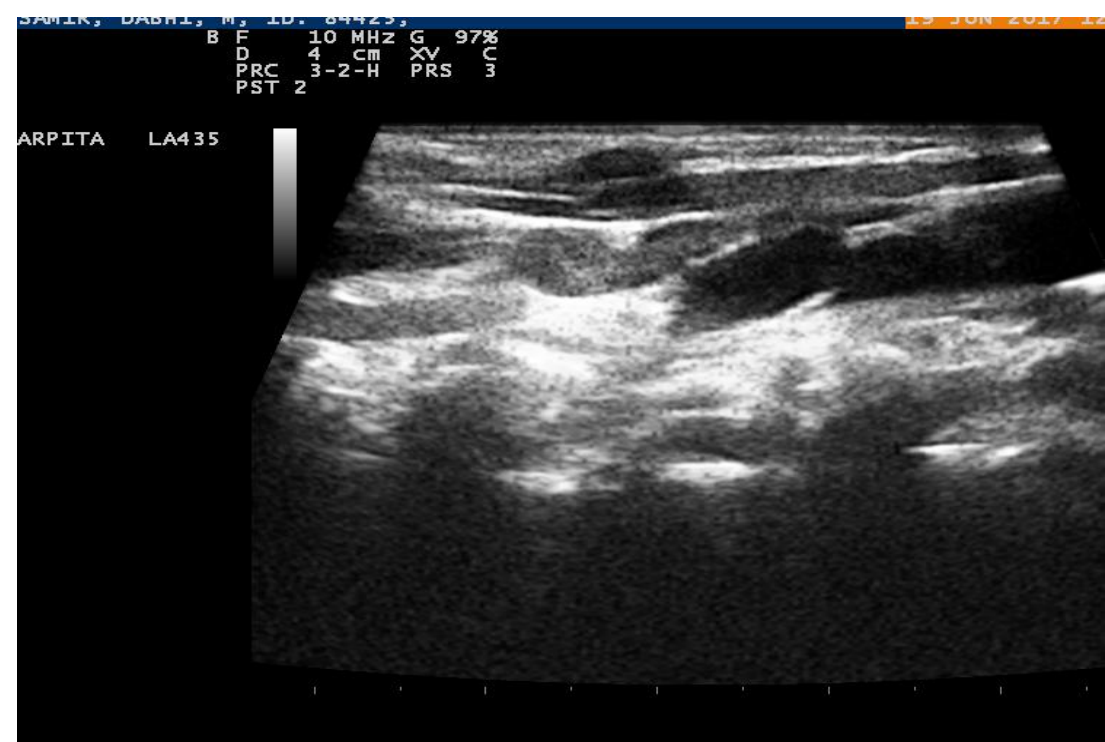

Figure 3: Multiple well defined hypoechoic lesions (reactive lymphnodes) seen in bilateral side of neck. FNAC proved final diagnosis.

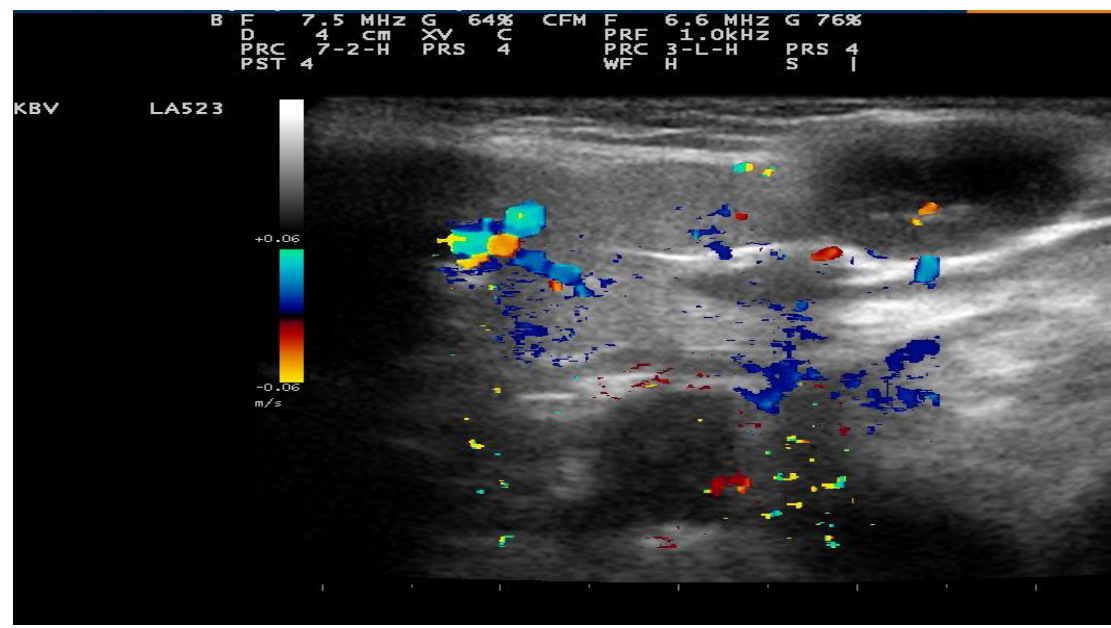

Figure 4: Multiple well defined hypoechoic lesions showing peripheral capsular vascularity . FNAC proved peripheral capsular vascularity . 


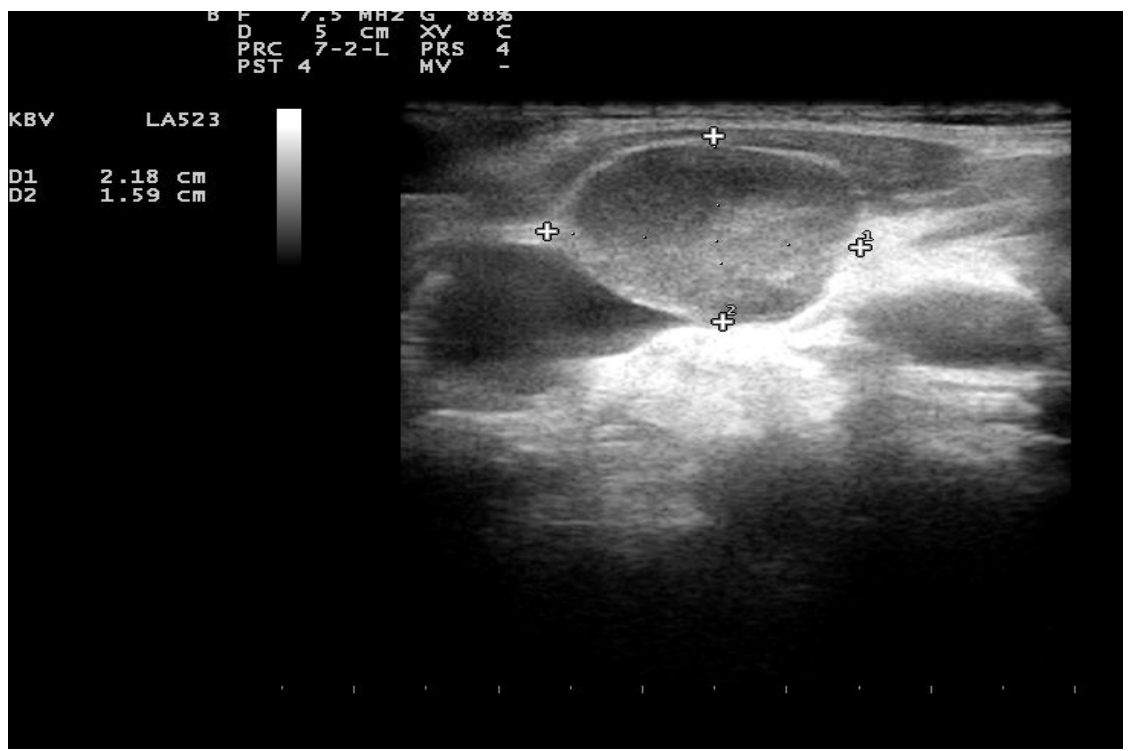

Figure 5: Well defined hypoechoic lesion (Malignant lymhnode) seen on right side of neck .

\section{References}

[1]. Ahuja AT, Ying M. Sonographic evaluation of cervical lymph nodes. AJR Am J Roentgenol 2005;184:1691-9. 2.

[2]. Takeuchi Y, Suzuki H, Omura K, Shigehara T, Yamashita T, Okumura K, et al. Differential diagnosis of cervical lymph nodes in head and neck cancer by ultrasonography. Auris Nasus Larynx 1999;26:331-

[3]. Haque MA, Talukder SI: Evaluation of fine needle aspiration cytology (FNAC) of lymphnodes in Mymensingh. Mymensingh Med J. 2003, 12 (11): 33-5.PubMedGoogle Scholar

[4]. Ying M, Ahuja A, Evans R: Cervical lymphadenopathy: sonographic differentiation between tuberculous nodes and nodal metastases from non-head and neck carcinomas. J Clin Ultrasound. 1998, 26: 383-389. 10.1002/(SICI)10970096(199810)26:8<383::AID-JCU2>3.0.CO;2-E.View ArticlePubMedGoogle Scholar

[5]. Asai S, Miyachi H, Suzuki K, Shimamura K, Ando Y: Ultrasonographic differentiation between tuberculous lymphadenitis and malignant lymph nodes. J Ultrasound Med. 2001, 20: 533-538.PubMedGoogle Scholar

[6]. Steinkamp HJ, Cornehl M, Hosten N, Pegios W, Vogl T, Felic R: Cervical lymphadenopathy: ratio of long to short axis diameter as a predictor of malignancy. Br J Radiol. 1995, 68 (807): 266-70. 10.1259/0007-1285-68-807-266.View ArticlePubMedGoogle Scholar

[7]. Kim HC, Han MH, Do KH, Kim KH, Choi HJ, Kim AY, Sung MW, Chang KH: Volume of cervical lymph nodes using 3 D ultrasonography. Differentiation of metastatic from reactive lymphadenopathy in primary head and neck malignancy. Acta Radiol. 2002, 43 (6): 571-4. 10.1034/j.1600-0455.2002.430606.x. View ArticlePubMedGoogle Scholar

[8]. Ying M, Ahuja A, Brook F: Accuracy of sonographic vascular features in differentiating different causes of cervical lymphadenopathy. Ultrasound Med Biol. 2004, 30 (4): 441-7. 10.1016/j.ultrasmedbio.2003.12.009.View ArticlePubMedGoogle Scholar

[9]. Ho SS, Metreweli C, Ahuja AT: Does anybody know how we should measure Doppler parameters in lymph nodes?. Clinical Radiology. 2001, 56: 124-126. 10.1053/crad.2000.0588. View ArticlePubMedGoogle Scholar

[10]. Mikami Y, Kamato S, Kawobata K, Nigaari T, Hoki K, Mitani H, Beppu T: Ultrasonographic evaluation of metastatic cervical lymph nodes in head and neck cancers. Nippon Jibiinkoka Gakkai Kaiho. 2000, 103 (7): 812-20.View ArticlePubMedGoogle Scholar

[11]. Danninger R, Posawetz W, Humer U, Stammberger H, Jakse R. Ultrasound investigation of cervical lymph node metastases: Conception and results of a histopathological exploration. Laryngorhinootologie 1999;78:144-9. 6.

[12]. Ahuja A, Ying M. Sonographic evaluation of cervical lymphadenopathy: Is power Doppler sonography routinely indicated? Ultrasound Med Biol 2003;29:353-9

[13]. Na DG, Lim HK, Byun HS, Kim HD, Ko YH, Baek JH. Differential diagnosis of cervical lymphadenopathy: Usefulness of color Doppler sonography. AJR Am J Roentgenol 1997;168:1311-6. 